\title{
Intramedullary Spinal Cord Sarcoidosis Mimicking Glioma by Magnetic Resonance Imaging: Case Report
}

\author{
R. A. Hauser, $M D,{ }^{1}$ S. L. Alden, $M D,{ }^{2}$ D. N. Coar, $M D,{ }^{3}$ M. R. Cundiff, \\ MD, ${ }^{4}$ C. C. Bundshuh, MD $^{5}$ \\ ${ }^{1}$ Department of Neurology, University of South Florida, Tampa, Florida, ${ }^{2}$ Department \\ of Physical Medicine and Rehabilitation, ${ }^{3}$ Department of Internal Medicine, ${ }^{4}$ Depart- \\ ment of Pathology, ${ }^{5}$ Department of Radiology, Eastern Virginia Graduate School of \\ Medicine, Medical College of Hampton Roads, Norfolk, Virginia, USA.
}

\section{Summary}

We present the case of a 31-year-old male who presented with a progressive myelopathy. Magnetic resonance imaging (MRI) demonstrated widening of the cervical and upper thoracic spinal cord and T2-weighted images demonstrated high signal intensity from the pontomedullary junction to the T6-T7 intervertebral level, consistent with the appearance of a spinal cord glioma. Although the patient had evidence of systemic sarcoidosis, a spinal cord biopsy was required to delineate the nature of the spinal cord lesion and dictate appropriate therapy.

Key words: Sarcoidosis; Intramedullary sarcoidosis; Magnetic resonance imaging; Myelopathy.

Sarcoidosis is a systemic non-caseating granulomatous disease of unknown aetiology. Neurological involvement occurs in approximately $5 \%$ of patients with sarcoidosis (Delaney, 1977; Stern, 1985). Spinal cord involvement is rare with only 19 cases reported by 1981 (Day, 1977; Cahill, 1981). We present the case of a patient with biopsy-proven intramedullary spinal cord sarcoidosis who presented with a progressive myelopathy. The MRI studies in this case were indistinguishable from those seen in cases of spinal cord gliomas.

\section{Case report}

A 31-year-old male was brought to the emergency room after experiencing a generalised motor seizure at home. The patient had a 17 year history of a seizure disorder and was non-compliant on phenytoin. He further related a history of the subacute onset and progression of weakness, worse in the lower than the upper extremities, over the previous 6 months. This weakness had accelerated over the 6 weeks prior to admission such that the patient could no longer ambulate. He reported occasional urinary incontinence but denied bowel or sexual dysfunction, sensory disturbance, back pain, headaches, shortness of breath or history of significant trauma. 
Physical examination revealed a blood pressure of $120 / 80 \mathrm{~mm} \mathrm{Hg}$ and a temperature of $37^{\circ}$. Heart sounds were of regular rate and rhythm. Lung, abdominal and genital examinations were normal. There was no lymphadenopathy. Skin examination was remarkable for moderately abundant 1 to $2 \mathrm{~mm}$ pale papules about the face, eyelids and neck. Neurological examination revealed the patient to be awake, alert and oriented. Cranial nerve examination was normal. Motor examination revealed weakness in all four extremities, greater in the lower than the upper extremities, worse on the left than the right and more pronounced distally than proximally. Muscle strength grading was as follows: right deltoid, biceps and brachioradialis $-4+$, triceps and wrist extensors- $3+$, wrist flexors and hand interossei-1+; left deltoid and biceps-3+, tricepts-2+, wrist flexors and hand intrinsics - 1+; right hip flexors, quadriceps, hamstrings and dorsi-. and plantar flexors$3+$, left hip flexors-3+, quadriceps and hamstrings-2+, and dorsi- and plantar flexors0 . Moderate atrophy of the intrinsic muscles of the hands was noted. Deep tendon reflexes were $1+$ in the upper extremities and $4+$ at the knees and ankles with sustained clonus. Plantar responses were extensor bilaterally. Pinprick appreciation was decreased from the C3 to T8 dermatomes. Proprioception was intact in the feet but diminished in the hands. Unsupported sitting balance was poor, and the patient was unable to stand without support.

The initial laboratory examination was remarkable for normal electrolyte, calcium, phosphorus and magnesium levels, a WBC count of $4.6 \times 10^{3} / \mathrm{UL}$, a haemoglobin of $12.8 \mathrm{~g} / \mathrm{dl}$, and a phenytoin level of less than $2.5 \mathrm{mcg} / \mathrm{ml}$. Arterial blood gases were normal. The admission chest X-ray revealed mild right paratracheal and bilateral hilar lymphadenopathy.

On the day following admission, the patient underwent MRI of the head and spine. This was performed using a high field strength (1.5-T) superconducting GE Signa system. A multislice, multiecho, intermediate, and T2-weighted spin echo sequence (SE 2200/20/90) was used. These studies demonstrated marked enlargement of the cervical and upper thoracic spinal cord. The T2-weighted images demonstrated a marked increase in signal intensity of the neuraxis from the pontomedullary junction to the T6-T7 intervertebral disc level (Fig. 1). The T1-weighted images demonstrated a slight decrease in the signal intensity in these areas. In addition, the MRI of the head demonstrated a $1 \mathrm{~cm}$ focal area of increased signal intensity in the medial right temporal lobe visualised best on the T2-weighted images. The MRI appearance of the brainstem/spinal cord lesion was thought to be consistent with an infiltrating glioma. The presence of both brain and spinal cord lesions suggested the possibility of a multicentric glioma or an infectious or inflammatory process.

Additional workup revealed an elevated angiotensin converting enzyme level of $63 \mathrm{u} / \mathrm{ml}$ (normal $=8-52 \mathrm{u} / \mathrm{ml}$ ). A PPD skin test was negative. Punch biopsy of a neck papule revealed non-caseating, granulomatous inflammation, consistent with sarcoidosis.

A decompressive laminectomy of C3 to C7 and spinal cord biopsy was performed. Cerebrospinal fluid obtained at the time of operation was clear and colourless with $0 \mathrm{RBC}$ and $48 \mathrm{WBC} / \mathrm{mm}^{3}$, a glucose level of $20 \mathrm{mg} / \mathrm{dl}$ and a protein level of $252 \mathrm{mg} / \mathrm{dl}$. CSF cultures and cytology were negative. Histological examination of biopsy specimens (Fig. 2) demonstrated infiltration of the spinal cord by non-caseating granulomas consisting of aggregates of epithelioid histiocytes with rare multinucleate giant cells. No areas of necrosis were identified. Acid fast and fungal stains were negative. The patient was placed on prednisone $80 \mathrm{mg} \mathrm{p.o.} \mathrm{daily} \mathrm{post-operatively} \mathrm{for} \mathrm{a} \mathrm{diagnosis} \mathrm{of} \mathrm{neurosarcoidosis.} \mathrm{There} \mathrm{was}$ minimal improvement in strength at 3 months follow-up.

\section{Discussion}

Spinal cord involvement in sarcoidosis has been noted to result in intramedullary, intradural-extramedullary, or extradural masses, granulomatous infiltration, or arachnoiditis (Day, 1977; Baruh, 1978; Karoff, 1980; Cahill, 1981). A recent review of the literature revealed only 24 cases of histologically confirmed spinal cord sarcoidosis (Hitchon, 1984).

In our case, the patient presented with seizures and a progressive myelopathy. His hilar adenopathy, elevated serum angiotensin converting enzyme level, and 


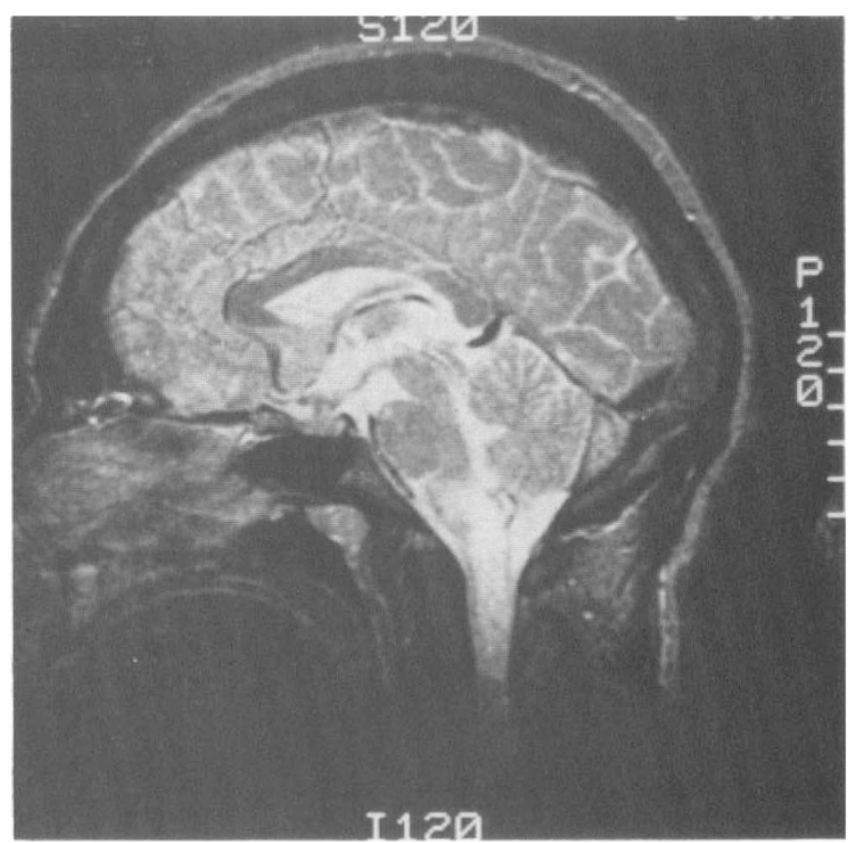

Figure 1 Spin echo T2-weighted (TR 2200, TE 90 msec.) sagittal MR image using a head coil demonstrates a diffuse increase in signal intensity in the entire medulla and visualised cervical spinal cord. Additional images (not shown) demonstrated a widened cervical spinal cord and increased signal intensity to the T6-T7 intervertebral level. T1-weighted images revealed a slight decrease in signal intensity.

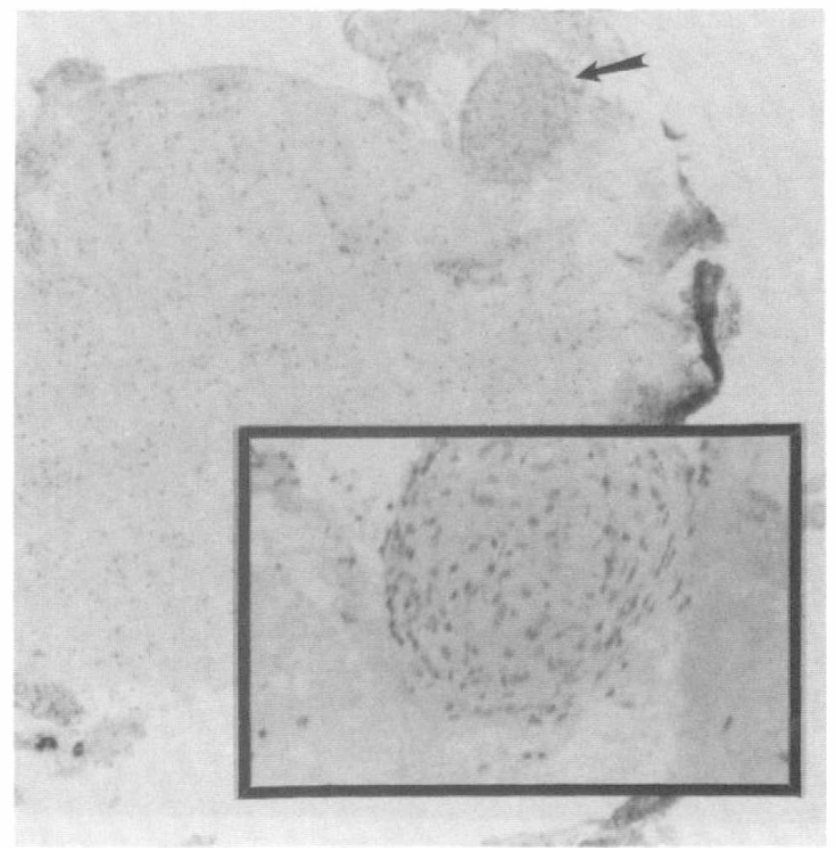

Figure 2 Spinal cord biopsy (magnification $=100 \times$ ) demonstrates an isolated, well-defined, nonnecrotising granuloma (arrow). Insert: $400 \times$ magnification of granuloma demonstrates epithelioid histiocytes. 
skin biopsy demonstrating non-caseating granulomas resulted in a diagnosis of systemic sarcoidosis. Skin lesions are seen in approximately $19 \%$ of patients with sarcoidosis (Olive, 1985). The most common types of skin lesions are maculopapules (42\%) and erythaema nodosum (39\%), although plaques and nodules are also encountered. This patient's abnormal CSF profile and intracranial lesion accompanying his spinal cord lesion suggested a diagnosis of neurosarcoidosis. However, prior to biopsy, it remained unclear as to whether his spinal cord lesion was due to sarcoidosis or a neoplastic process. MRI clearly delineated the intramedullary location and extent of the lesion, but not its nature.

Kelly et al. (1988) recently reported the case of a 52-year-old woman who presented with the complaint of tingling sensations and pain in her neck and all four extremities for several months. Physical examination revealed extreme pain and limitation of movement of the cervical spine. She had no evidence of systemic sarcoidosis. T1-weighted MR images of the cervical spine demonstrated only widening of the cord. T2-weighted images demonstrated a diffuse increase in signal intensity extending from the lower medulla to approximately $\mathrm{T} 3$, thought to most likely represent an infiltrating glioma. However, the spinal cord biopsy of this lesion was consistent with neurosarcoidosis. The MR images in our case and that of Kelly et al. are remarkably similar. In each, T1-weighted images demonstrated widening of the cord (and in our case a subtle decrease in signal intensity), and the T2-weighted images revealed an increase in signal intensity from the medulla to the thoracic region. Clinically, our patient had evidence of systemic sarcoidosis, while that of Kelly et al. did not. The MRIs alone were non-specific as to aetiology, and could not differentiate neurosarcoidosis from glioma.

When presented with this MRI picture, the clinician must consider sarcoidosis in the differential diagnosis, and undertake a search for evidence of systemic sarcoidosis. Whether evidence of systemic sarcoidosis is present or not, a biopsy of the spinal cord lesion is required to define the aetiology of the lesion and dictate appropriate therapy.

\section{References}

Baruh JK, Glasauer F, Ranajit S, Smith B 1978 Sarcoidosis of the cervical spinal canal: case report. Neurosurgery 3:216-218

CAhill DW, SAlCMAN M 1981 Neurosarcoidosis: A review of the rarer manifestations. Surgical Neurology 15:204-211

DAY A, SYPERT G 1977 Spinal cord sarcoidosis. Annals of Neurology 1:79-85

DELANEY P 1977 Neurologic manifestations in sarcoidosis: Review of the literature with a report of 23 cases. Annals of Internal Medicine 87:336-345

Hitchon PW, Ul HaQue A, Olson JJ, JaCOBS SK, Olson SP 1984 Sarcoidosis presenting as an intramedullary spinal cord mass. Neurosurgery 15:86-90

KANOFF R, RUBURG R 1980 Sarcoidosis presenting as a dorsal spinal cord tumor: Report of a case. Foumal of the American Osteopathic Association 79:765-767

Kelly RB, MAHONY PD, CARVLEZ KM 1988 MR demonstration of spinal cord sarcoidosis: Report of a case. AfNR 9:197-199

Olive K, Kataria Y 1985 Cutaneous manifestations of sarcoidosis. Relationships to other organ system involvement, abnormal laboratory measurements, and disease course. Archives of Internal Medicine 145:1811-1814

Stern BJ, Krumholz A, Johns C, Scott P, Nissim J 1985 Sarcoidosis and its neurological manifestations. Archives of Neurology 42:909-917 